\title{
Emerald COMPEL
}

\section{Study for performance characteristics of surface permanent magnet motor at various magnetization patterns}

\begin{tabular}{|r|l|}
\hline Journal: & $\begin{array}{l}\text { COMPEL: The International Journal for Computation and Mathematics in } \\
\text { Electrical and Electronic Engineering }\end{array}$ \\
\hline Manuscript ID & COMPEL-03-2016-0114 \\
\hline Manuscript Type: & Original Article \\
\hline Keywords: & $\begin{array}{l}\text { Surface permanent magnet (SPM) motor, Magnetization pattern, Halbach } \\
\text { magnetization array, Electromagnetic characteristics, Electromechanical } \\
\text { characteristics }\end{array}$ \\
\hline
\end{tabular}

SCHOLARONE

Manuscripts 


\title{
Study for performance characteristics of surface permanent magnet motor at various magnetization patterns
}

\begin{abstract}
Purpose - In the present paper the influence of magnetization patterns of the rotor magnets on the performance characteristics for surface permanent magnet (SPM) motor is investigated. The objective is to show how the electromagnetic and electromechanical characteristics of this type of motor can be significantly changed by applying various magnetization patterns of permanent magnets placed on the rotor surface.

Design/methodology/approach - At the beginning a survey of possible and most frequently used magnetization patterns for permanent magnet motors is presented. The research is focussed on the comparison of performance characteristics and is developed into three levels. It is started with a conventional SPM motor having segmented permanent magnets, and two magnetization patterns are considered: parallel and radial. Showing no significant difference of the motor performance at parallel and radial magnetization, for further investigation only radial magnetization, being more conventional, is considered. In the second step, the counterparts of SPM with two Halbach array configurations, under the constraint of fixed magnet volume, are studied. Finally, detailed comparative analyses of SPM at radial, Halbach 1 and Halbach 2 magnet patterns are presented. Advantages and drawbacks for suggested magnet configurations are discussed.

Findings - It is presented how the magnetization pattern of the rotor permanent magnets can have a substantial impact on the SPM motor performance characteristics. From the analysis of magnetic field properties at various types of magnetization, it is observed that both shape and rates of the characteristics, for radial magnetization and Halbach 2 configuration, exhibit similar features. It is due to the fact that Halbach 2 array cancels magnetic flux above the permanent magnets, i.e. strengthens the magnetic field in the rotor, and enhances the coupling between the rotor and stator magnetic field. It is worth to emphasise, that due to less saturation of the magnetic core and lower iron loss at Halbach 1 and Halbach 2 magnetization, it is possible to increase armature current and consequently to increase the electromagnetic torque. This fact could be interesting for further research.

Originality/value - The paper presents an original comparative analysis for performance characteristics of a surface permanent motor at various magnetization patterns. The novelty of the paper is seen in the introduction of two Halbach magnetization array for permanent magnets and improvement of the performance characteristics for the analysed motor.
\end{abstract}

Keywords - Surface permanent magnet (SPM) motor, Magnetization pattern, Halbach magnetization array, Electromagnetic characteristics, Electromechanical characteristics.

Paper type - Research paper

\section{$\underline{\text { Introduction }}$}

The demand for more compact and more effective electric machines attracted the attention of researchers and manufacturers towards the permanent magnet machines. There is an evident tendency the conventional motors to be replaced by their permanent magnet counterparts with special structures that offer new features, making them more attractive for use in electric drive systems. In fact, the permanent magnet motors (PMMs) are more and more recommended and used in the industrial applications, mainly due to the increased power density, higher efficiency, better dynamic performance and higher values of torque per unit volume. Knowing that their design is simpler with increased life time and easier maintenance, the advantages of PM motors are evident. The new generation of high performance permanent magnets that are capable to provide strong enough magnetic fields has expanded the application range of magnets to electric motor topology and design.

A Halbach magnet array is a specific arrangement of permanent magnets. In this structure the magnetic field on one side of the array is augmented while on the other side is almost cancelled. This novel magnet pattern is commonly called Halbach array. The Halbach array has been applied to various magnet systems for industrial applications: from magnetic bearings, brushless motors and magnetically levitating (maglev) 
systems, to high-tech applications such as wiggler magnets of particle accelerators as well as nuclear magnetic resonance (NMR) devices.

Many authors have presented numerous arrangements, design and analyses of applications for Halbach PM arrays: Shute et al. (2000) presented an extensive mathematical analysis of one-sided fluxes in various magnetized structures. A very powerful and fast analytical method to compute Halbach magnetic interactions presented Allag et al. (2009), while Rovers et al. (2009) determine static forces induced in Halbach arrays. Choi and Yoo (2008) determine the number of layers in Halbach magnets and Cha et al. (2008) study topology of eddy current couplings and brakes with Halbach magnets.

The general question is when and why one should use a Halbach PM array in the design of surface mounted permanent magnet motors? It is known that electric motors with Halbach PM arrays hold several attractive features, as: almost sinusoidal airgap field distribution and back-emf waveform, strong field intensity, low cogging torque, potentially high airgap flux density and sometimes there is no need of rotor back-iron. In one of the first works in this area (Marinescu and Marinescu, 1992) the authors presented the new concept of PM excitation, using Halbach magnetization pattern. The analysis of the torque production capabilities of Halbach and conventional magnet arrays under the constraints of fixed magnet volume presented Ofori-Tenkorang and Lang (1995). Halbach magnetized surface permanent magnet (SPM) motors are continuously attracting much research and development interest resulting in extensive investigation for their design topologies and applications. Nowadays a lot of research work in potentials of Halbach array is done by Mansson $(2014,2015)$.

By using finite element method Fan and Wu (2012) optimise the Halbach magnet in permanent magnet synchronous motor. On the other hand, Zhu et al., (2002) compared the characteristics of brushless machines with discrete magnet segments and a single ring magnet. Kataoka et al. (2013) presented the analysis of three magnet array-type rotors in Vernier motor, while Winter et al. (2012) studied the shape of Halbach array in axial flux motors. Mainly, the analyses of performance characteristics and features for variety of devices using Halbach magnet arrays are done by FEA (Livadaru et al., 2011; Ibtissam et al., 2014; Jian and Chau, 2010). A procedure for minimising torque pulsations in Halbach array permanent magnet machines propose Sadeghi and Parsa (2012).

High-performance electric drives require smooth static electromagnetic torque, high peak-torque at rather low component of cogging torque. In the Surface Permanent Magnet (SPM) motors, the torque is caused by the interaction between rotor permanent magnet field and stator armature reaction field. Consequently, the magnetization pattern of the rotor magnets has an important influence on the torque characteristics of the motor. To get the best results we need to enhance this interaction.

In the paper we analyse how the magnetization pattern affects the magnetic field and torque production in surface permanent magnet (SPM) motors. The presented study has a comparative character, between conventional surface PM synchronous motor, and its counterparts designed with two modifications of Halbach magnet array configuration. Thus, the influence of magnet configuration on performance of surface mounted permanent magnet motors is analysed. Along with conventional magnetization of permanent magnets, two possible Halbach magnetizing patterns produced by discrete arrangement of magnet segments, are considered. The electromagnetic and electromechanical characteristics, when such magnetization patterns are employed in a surface permanent magnet motor, are determined and analysed. A comparison between performance characteristics of a conventional surface PM motor and two Halbach array topologies is presented. The study is consisted of FEM based numerical experiments that put in view properties of the static electromagnetic and cogging torque characteristics. In addition, electromagnetic features, such as electromagnetic field distribution in the motor, magnetic flux per pole and components of the flux density in the air gap, are also analysed.

\section{Halbach Magnetization Patterns}

Several rotor topologies for permanent magnet machines are possible. This variety depends on the shape of permanent magnets and their arrangement in the rotor. Most PMMs use permanents magnets which are mounted on the rotor surface, but they can be also inset magnets, interior magnets or buried magnets. The effect of position of permanent magnets as well as their magnetization patterns on the machine performance characteristics are significant.

In conventional surface permanent magnet motors, the magnet pole parts can be magnetized with constant direction - parallel magnetization, or with a slightly different magnetization direction depending on their 
position within the pole arc - radial magnetization. Halbach magnetized PM machines are novel and they offer many new and attractive features.

All it started back to the year 1973 when it was realized that when a planar structure, such as flat plate or disc, has varying in-plane and out-of-plane components of magnetization, then all the external fringing flux emerges below the structure, with identically zero flux appearing above. In a published paper (Mallinson, 1973 ) it was shown previously unknown class of magnetization patterns that exist in planar structures with the unique property such that all the flux escapes from one surface with none leaving the other side. At the time, his discovery was considered as a "magnetic curiosity".

Almost simultaneously and independently, Halbach made a closely related invention, showing that, by assembling blocks of uniformly magnetized permanent magnets, both for two-dimensional and threedimensional structures, could be produced significantly increased fringing flux. In consequence, few years after, it was reported a novel PM configuration (Halbach, 1980), which later has been denoted by the name of its inventor. A Halbach array is a special arrangement of permanent magnets that augments the magnetic field on one side of the array while almost cancelling the field on the other side. This is achieved by a spatially rotating pattern of linear magnetisation. A continuously varying magnetization pattern, as presented in Figure 1 (a), yields truly one-sided fluxes. The ideal linear Halbach array has pure sinus magnetic profile, on the enhanced side of the array, while cancelling the field on the other side. The practical Halbach array is shown in Figure 1 (b).

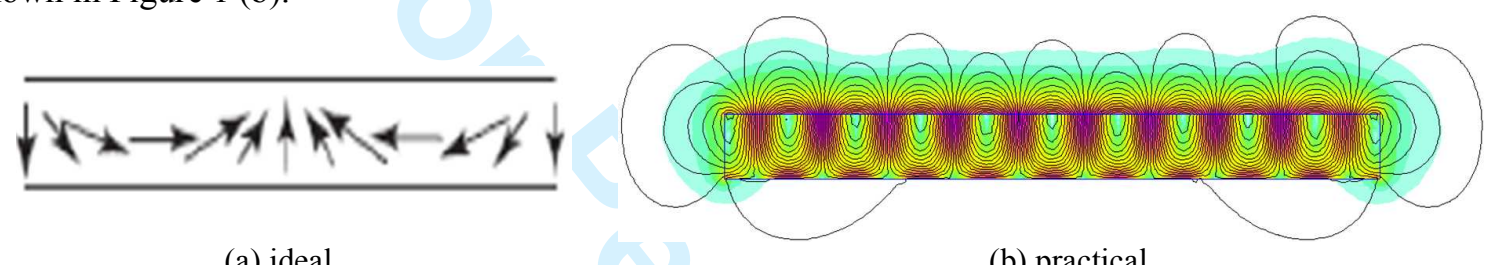

(a) ideal

(b) practical

Figure 1. Illustration for linear Halbach array

The idea of such self-shielding property did not immediately gain widespread acceptance because of the difficulty in realization. Instead, an array of rectangular or square permanent magnets (PM) is actually used. These non-ideal Halbach arrays do not provide a purely sinusoidal magnetic field on the enhanced side and a zero magnetic field on the cancelled side; however, they still provide much better performance characteristics than simply using an array composed of alternating polarity magnets, magnetized by conventional patterns - parallel or radial.

An approximated continuously varying magnetization array is achieved by using a number of segmented magnets with varied magnetization angles; such novel construction of permanent magnet poles is named Halbach magnetization or Halbach array hereafter. Basically, the topology has magnet segments with distinct magnetization direction, presented in Figure 2 (a) when magnetic flux is cancelled below the magnets - adopted as Halbach 1, and Figure 2 (b) for the magnetic field cancelled above the permanent magnets - adopted as Halbach 2.

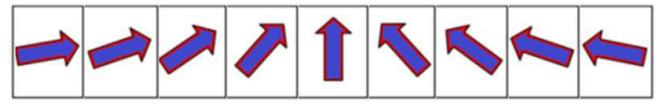

N-pol

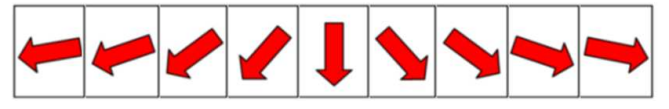

S-pol

(a) Halbach array 1: magnetic field is cancelled below the permanent magnets

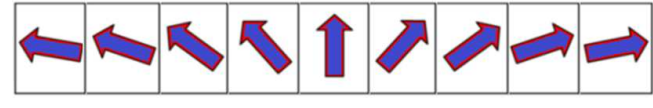

N-pol

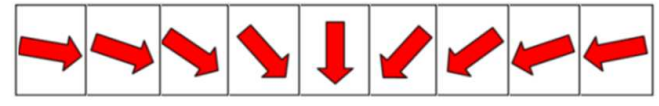

S-pol

(b) Halbach array 2: magnetic field is cancelled above the permanent magnets

Figure 2. Practical nine-piece Halbach array magnetization patterns for pair of poles 
Although promising, the Halbach's research results, mainly due to linear layout of the magnets, were not seen as possible solution for rotating electrical machines. But it was only a matter of time till the radial design was proposed and was successfully implemented for electric motors design. To date, Halbach magnetized machines have generally been fabricated from premagnetized magnet segments with the appropriate magnetization orientations, which approximate the Halbach array. In addition, the existence of self-shielded flux in the magnet poles, sometimes could lead to that rotor back-iron can be even abandoned.

\section{Case Study}

The analysis model is a surface permanent magnet motor with $18 \mathrm{~A}$ rated current, 0-10 Nm torque control, and $0-4000 \mathrm{rpm}$ speed control. Both rotor and stator are iron cored structures. The stator lamination is with 36 slots, where 3 single-layer stage windings are placed. The drive is connected to a current-source inverter and is controlled by rectangular current waveforms. At each instant of time one winding is energised by a positive current wave $+\mathrm{I}$, the other by negative $-\mathrm{I}$, while the third winding remains unexcited.

There are 6 surface mounted segmented SmCo5 magnets on the rotor. The pole arc is $54^{0}$ with 9 segments arranged in radial direction and 15 layers in $90 \mathrm{~mm}$ axial length. This segmented PM structure is convenient to investigate how various magnetization patterns effect the magnetic field distribution, torque production and performance characteristics of SPM motor. Four different magnetization patterns, as presented in Figure 3 have been considered, and the respective performance characteristics are compared: (a) parallel magnetization - with constant direction of the magnetic flux; (b) radial magnetization - flux is directly pointed normally across the air gap; (c) Halbach 1 array - magnetic flux is cancelled in the rotor core, i.e. below the permanent magnets; (d) Halbach 2 array - magnetic flux is cancelled in the stator teeth, i.e. above the permanent magnets.

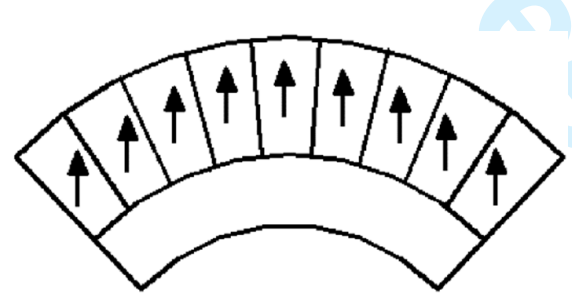

(a) parallel magnetization

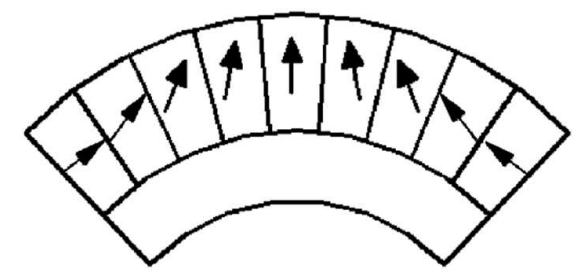

(c) Halbach 1 array

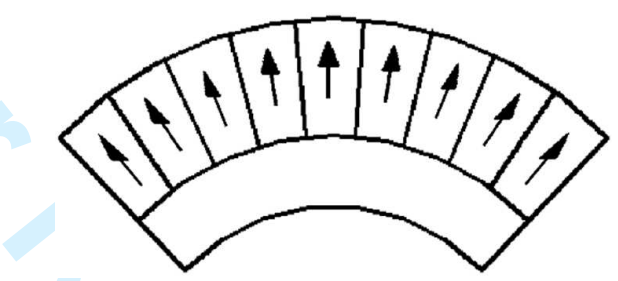

(b) radial magnetization

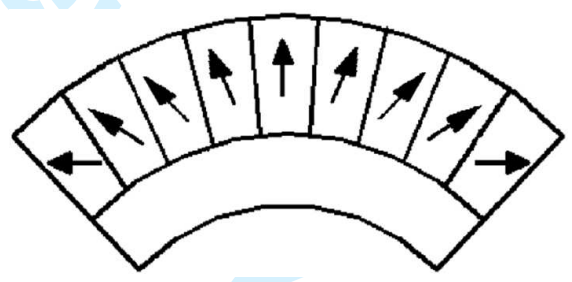

(d) Halbach 2 array

Figure 3. Four analysed magnetization patterns: N-pol is depicted

The study presented in this paper is mainly a simulation one. The analysis tool is software based on the finite element method, which will enable to investigate the magnetic field properties in the studied SPM motor, as well as the derived electromagnetic and electromechanical quantities and characteristics. Usually, there are different tools that can be employed in numerical simulations and they depend on the state of the analyzed system, i.e. steady state or transient operation. We intend to focus on the properties of the magnetic field created by permanent magnets; hence the magnetostatic analysis is relevant. It has to be pointed out that this FEM approach catches a given moment of the motor operation. Consequently, rotation, speed or voltage/current variations are not considered; they are represented by scalar values corresponding to the particular moment that is analyzed.

Since the presented research has a comparative character, it is developed into three levels. It is started with a conventional SPM synchronous motor with segmented permanent magnets; two magnetization patterns are considered: parallel and radial, as presented in Figure 3 (a) and (b). After, the counterparts with 
two Halbach array configurations are studied: Halbach 1, and Halbach 2, that are shown in Figure 3 (c) and (d), respectively. The FEM calculations are performed when only the permanent magnets generate the magnetic field. A part of the magnetic field distribution spanned to an N-pole pitch is shown in Figure 4 (a) to $(\mathrm{d})$.

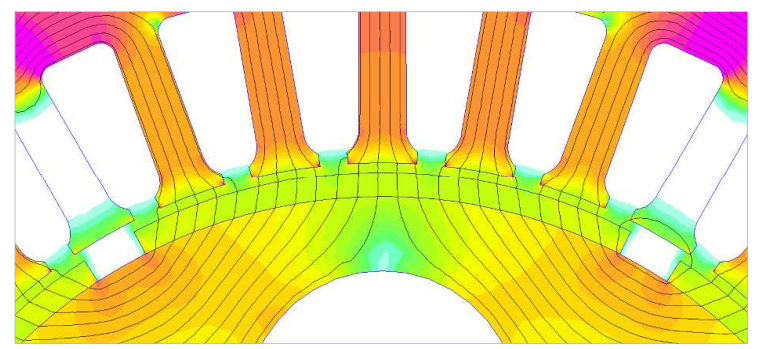

(a) parallel magnetization

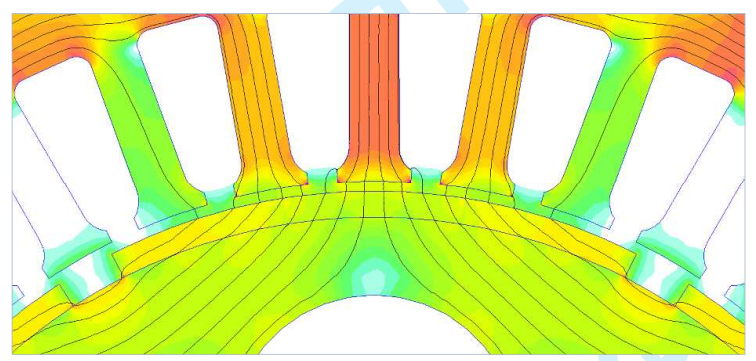

(c) Halbach 1 array

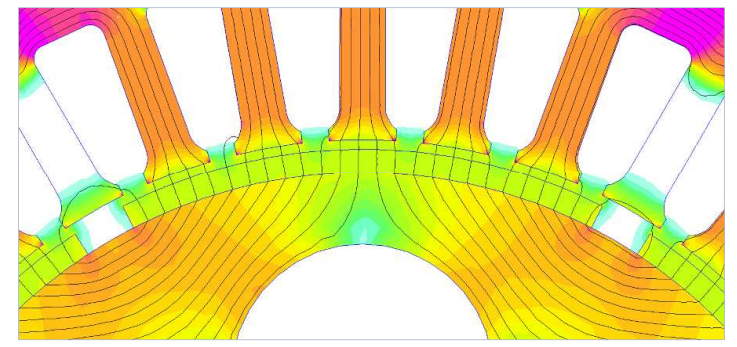

(b) radial magnetization

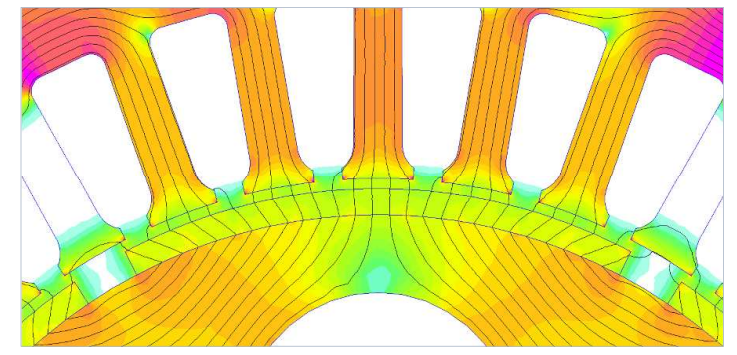

(d) Halbach 2 array

Figure 4. Magnetic field distribution for analysed PM magnetization patterns: no-load at $\theta=0^{0}$ rotor position

The results have proved that there is no significant difference between the motor characteristics at parallel and radial magnetization of the permanent magnets. As an example can serve the maximum value of magnetic flux per pole, which is for parallel $2.24 \mathrm{mVs}$, while for radial magnetization it is $2.31 \mathrm{mVs}$, i.e. $\sim 3 \%$ difference; at the same time, the profiles of their characteristics $\Phi_{p}=\mathrm{f}(\theta)$, as shown in Figure 5 are almost identical.

Consequently, only radial as more common, Halbach 1 and Halbach 2 magnetizations will be analysed.

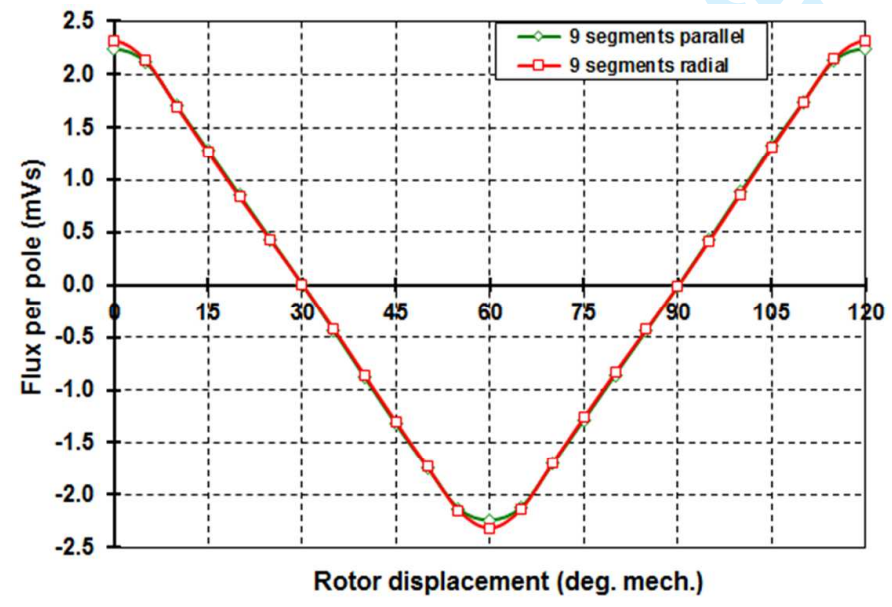

Figure 5. Flux characteristics $\Phi_{p}=\mathrm{f}(\theta)$ for conventional magnetizations (parallel and radial) at no-load 
Taking into consideration the radial magnetization and two Halbach arrays the performance analysis of the SPM motor is carried out. It is important to emphasise, that due to less saturation of the magnetic core and lower iron loss at Halbach 1 and Halbach 2 magnetization array, it is possible to increase the armature current and consequently to increase the electromagnetic torque, which in turn will give the advantage to the SPM motors with Halbach type array magnetization.

\section{Computational Results}

For prediction the performance characteristics of the studied SPM motor, the 2D Finite Element Analysis (FEA) is employed. The mesh of finite elements is with more than 123,000 nodes and 245,000 elements. The selection of the relevant results is presented in the subsequent headings.

\section{$\underline{\text { Magnetic Field Analysis }}$}

In Figure 6 is presented the magnetic flux density colour map and flux lines at rated current $I_{n}=18 \mathrm{~A}$ and for rotor position $\theta=30^{\circ} \mathrm{mech} .=90^{\circ} \mathrm{el}$., for the analysed magnetization types of rotor magnets.

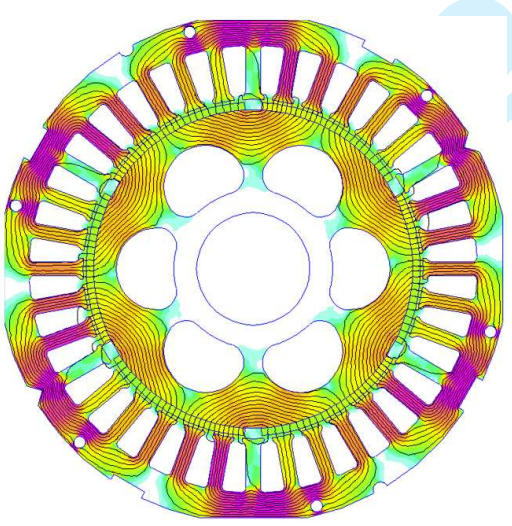

(a) radial magnetization: 27 lines

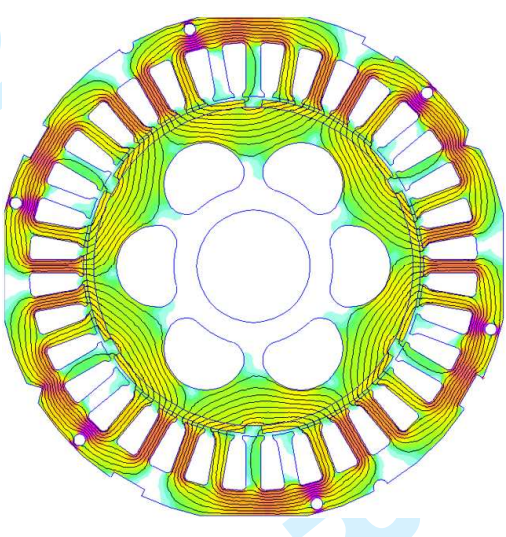

(b) Halbach 1 array: 19 lines

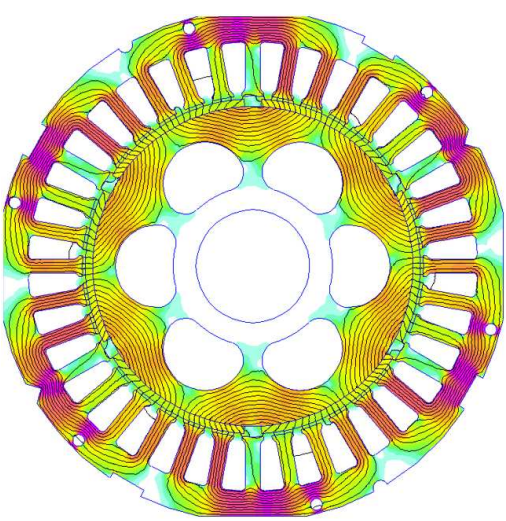

(c) Halbach 2 array: 25 lines

Figure 6. Magnetic field plots at rated load $I_{n}=18 \mathrm{~A}$ and rotor position $\theta=90^{\circ}$ el.

In order to get comparable views for all figures, the maximum value of the flux density is set on $1.7 \mathrm{~T}$, while the number of flux lines is proportional to the respective value for the magnetic vector potential $A_{\max }$ $(\mathrm{V} / \mathrm{s})$. Thus, one can observe the saturation level and weak regions for the analysed magnetization patterns. A part of more interesting results is presented in Table 1.

Table 1 Magnetic field properties of SPM motor at various magnetization patterns

\begin{tabular}{|l|c|c|c|c|c|c|c|}
\hline \multirow{2}{*}{ Description } & \multirow{2}{*}{ Unit } & \multicolumn{3}{|c|}{ No-load $I=0 ; \theta=0^{0}$} & \multicolumn{3}{c|}{ Rated load $I_{n}=18 \mathrm{~A} ; \theta=0^{0}$} \\
\cline { 3 - 8 } & & Radial & Halbach 1 & Halbach 2 & Radial & Halbach 1 & Halbach 2 \\
\hline $\begin{array}{l}\text { Magnetic vector } \\
\text { potential } A_{\max }\end{array}$ & $\mathrm{V} / \mathrm{s}$ & 0.01334 & 0.00854 & 0.01264 & 0.01496 & 0.01103 & 0.01453 \\
\hline $\begin{array}{l}\text { Magnetic flux } \\
\text { per pole } \Phi_{p}\end{array}$ & $\mathrm{mVs}$ & 2.31405 & 1.33240 & 2.14514 & 2.59865 & 1.80858 & 2.45885 \\
\hline $\begin{array}{l}\text { Magnetic flux } \\
\text { density } B_{m}\end{array}$ & $\mathrm{~T}$ & 0.55052 & 0.34437 & 0.51033 & 0.61823 & 0.43027 & 0.58497 \\
\hline
\end{tabular}

\section{Electromagnetic Characteristics}

In Figure 7 are presented the characteristics of the air-gap flux per pole $\Phi_{p}=\mathrm{f}(\theta)$ at no-load (a) and at rated load $I_{n}=18 \mathrm{~A}(\mathrm{~b})$, respectively; as it can be expected Halbach 1 array generates lowest magnetic flux. 


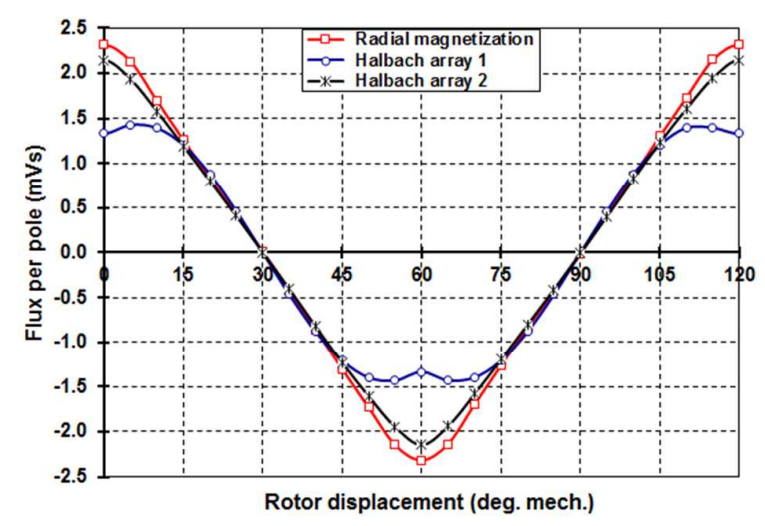

(a) no-load $I=0$

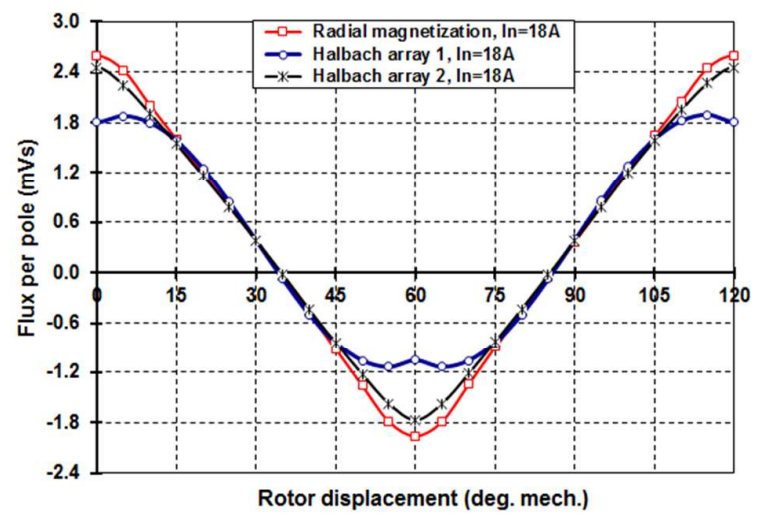

(b) rated load $I_{n}=18 \mathrm{~A}$

Figure 7. Comparative characteristics of the magnetic flux per pole $\Phi_{p}=\mathrm{f}(\theta)$

The spatial distribution of the magnetic flux density components, along the mid-gap line, is shown comparatively in Figure 8, for radial component (a) and for tangential component (b); the results are calculated when the magnetic field is generated by the permanent magnets only, i.e. for $I=0$.

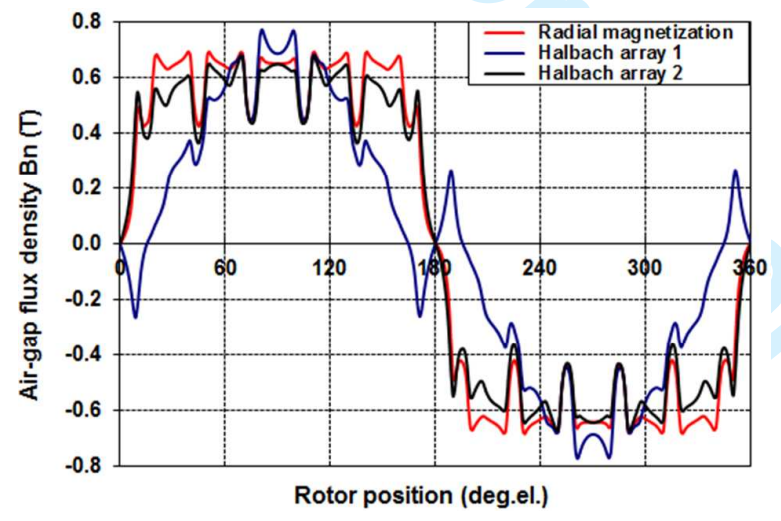

(a) normal component $B_{n}=\mathrm{f}(\theta)$

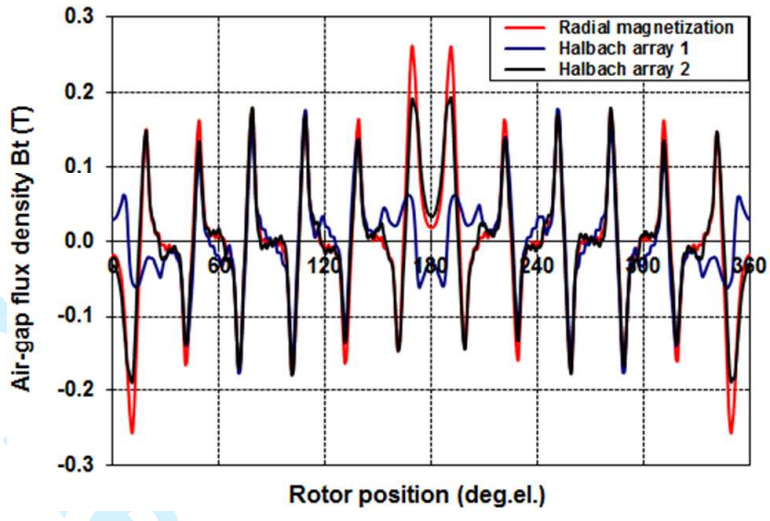

(b) tangential component $B_{t}=\mathrm{f}(\theta)$

Figure 8. Comparative characteristics of magnetic flux density in dependence of rotor position, at no-load

\section{Electromechanical characteristics}

In the study, the stress is put on the cogging and static torque characteristics. The weighted stress tensor volume integral is used to calculate the torques. This approach greatly simplifies the computation of torques and gives the most accurate results, as compared to line integration to calculate Maxwell stress tensor, or to application of energy concept and numerical differentiation of the air-gap magnetic coenergy. In order to achieve closer estimation of torques, the mesh density in the air gap, where the Maxwell stress is the highest, is particularly refined (Petkovska et al., 2015). The FEM simulations start at no-load, without current in stator windings, when the magnetic field is induced only by the permanent magnets and the cogging torque is computed. For the static torque to be predicted, field computations continue at rated load operation.

\section{$\underline{\text { Cogging torque }}$}

Cogging torque $T_{c o g}$ is probably the most annoying parasitic element in PM motors, because it is an undesired motor output, produced due to the interaction between the rotor magnets poles and slots of the stator, i.e. the stator saliency. The cogging torque is calculated at no-load, for all magnetization patterns, while rotor is displacing along one pole pitch in clockwise direction, i.e. for $60^{0}$ mech., with a step of $1^{0}$ mech. The cogging torque profile $T_{\operatorname{cog}}=\mathrm{f}(\theta)$ is depicted in Figure 9. 
Parallel and radial magnetization do not show significant discrepancy. Halbach 2 array exhibit similar profile as radial magnetization, while Halbach 1 array is more favourable, as peak-value is four times lower although at doubled frequency. In Figure 10 are displayed the cogging torque characteristics comparatively, following the same way as previous and omitting the parallel magnetization pattern.
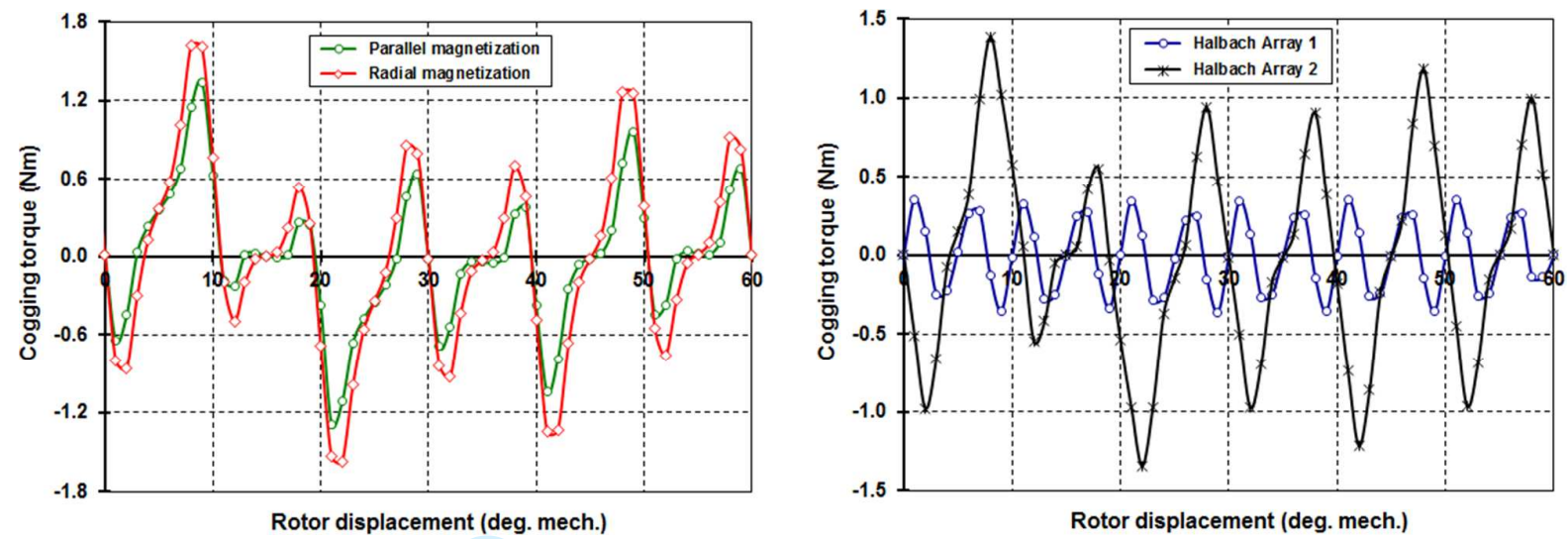

Figure 9. Cogging torque characteristics at different PM magnetization patterns

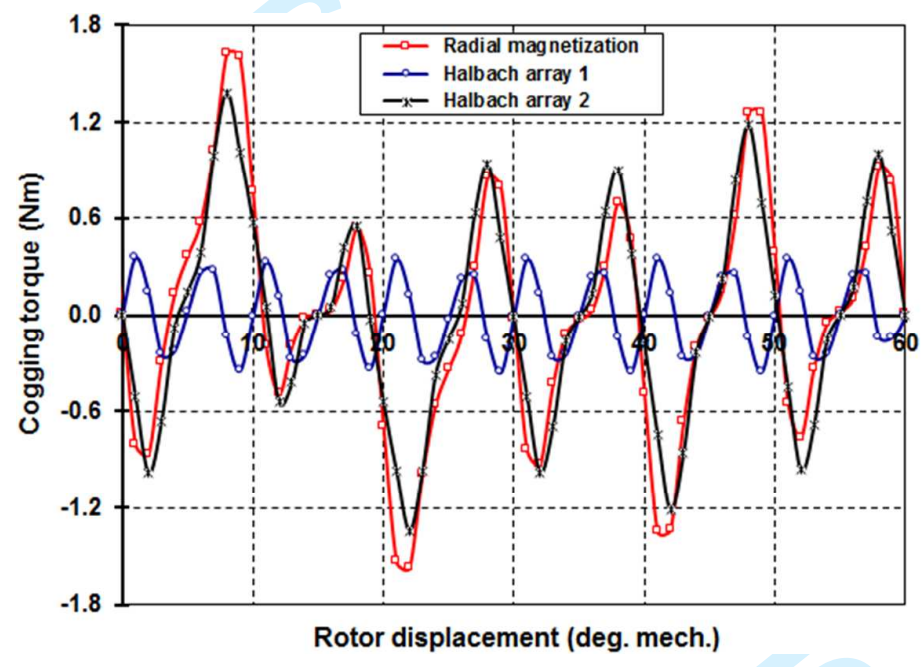

Figure 10. Comparative cogging torque characteristics

\section{$\underline{\text { Static torque }}$}

To evaluate the operating performance of surface permanent magnet synchronous motor at loading conditions with different magnetization patterns, it is required to analyse torque production and respective static torque characteristics. Electromagnetic torque is caused by interaction between rotor permanent magnets and stator armature reaction field. All four different magnetization patterns are again analysed.

The rated operating mode is simulated by setting in the stator windings rectangular current waves, as follows: $I_{A}=+I_{n}, I_{B}=-I_{n}$, while the phase winding $\mathrm{C}$, remains no energised $I_{C}=0$. Such current distribution enables to use 2D magnetostatic field simulations. Starting with the initial position when rotor and stator field are aligned, rotor is displaced for $5^{0}$ mech. in clockwise direction.

The static electromagnetic torque is computed and the comparative characteristics $T_{e m}=\mathrm{f}(\theta)$ for the analysed magnetization patterns, spanned along one full period of $120^{\circ}$ mech., i.e. $360^{\circ}$ el., are given in Figure 11. The computational results prove again that parallel and radial magnetization characteristics are very close and hence, as mentioned before, only the characteristic for radial magnetization have been considered. The emphasis is put on the Halbach magnetization patterns - Halbach 1 and Halbach 2 arrays. 


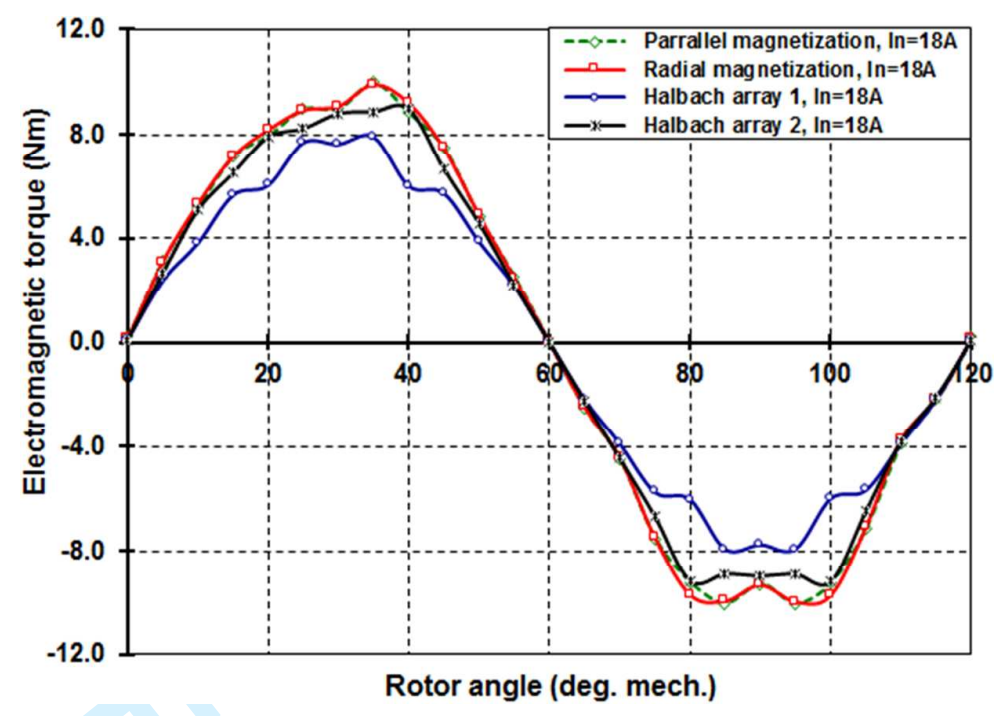

Figure 11. Comparative static torque characteristics $T_{e m}=\mathrm{f}(\theta)$ at rated load $I_{n}=18 \mathrm{~A}$

From the static and cogging torque characteristics it is evident that Halbach 1 array exhibits the symmetrical profile, but with the lowest rate of torque values with the biggest ripple. However, the advantage of this magnetization pattern is seen in the lowest cogging peak-torque, as seen in Figure 10. But it should be emphasized that the drawback is doubled frequency of its change; namely, instead of six, there are twelve periodical changes of the profile. On the other hand, Halbach 2 array pattern prevails radial magnetization because of the lower level of ripples, lower cogging torque for $\sim 15 \%$, and equal torque at rated operating load angles $7^{0}-10^{0}$ deg. mech. The only drawback is slightly lower peak static torque. However, due to less saturated stator teeth zone and lower iron loss, it is possible to increase the current in the stator windings; respectively the static electromagnetic torque will be increased and accordingly, this disadvantage will be compensated.

\section{Conclusions}

In the paper the influence of magnetization patterns on the performance characteristics for surface permanent magnet motor has been analysed. The computational results obtained by 2D magnetostatic FEM are used for detailed performance analyses. Four different magnetization patterns have been considered: two conventional - parallel and radial, and two recently developed configurations - Halbach 1 and Halbach 2 arrays. The particular emphasis has been put on torque characteristics. In addition, the electromagnetic features, such as electromagnetic field distribution of the motor, magnetic flux per pole and components of the flux density in the air gap, are also analysed.

From the analysis of FEM computational results and a review of the magnetic field properties at studied magnetization patterns, one can conclude that both the values and shape of the characteristics, for the radial magnetization and Halbach 2 configuration, exhibit similar features. It is due to the fact that Halbach 2 array cancels magnetic flux above the permanent magnets, i.e. strengthens the magnetic field in the rotor, and enhances the coupling between the rotor and stator magnetic field. On the other hand, Halbach 1 array exhibits weakened magnetic field, and consequently less saturated machine with not enough effective use of the active materials, which in turns leads to lower electromagnetic torque values with higher ripples; this fact has been confirmed with respective characteristics and diagrams. It should be pointed out that in the case of Halbach 1 array the cogging torque is with the lowest value.

It is worth to emphasise, that due to less saturation of the stator magnetic core and lower iron loss both at Halbach 1 and Halbach 2 magnetization array, it is possible to increase the armature windings current and thus to increase the electromagnetic torque, which in turn will give the advantage to the SPM motor with Halbach arrays magnetization. This fact could be interesting for further analyses.

The next task will be to analyse the induced back-EMF waveforms at different magnetization patterns of permanent magnets. An analysis of the losses in permanent magnets, as well as overall losses and efficiency 
of the SPM motor, by using the time-stepping finite element method could also be of interest. This work would serve as a good guide.

$\underline{\text { References }}$

Allag, H., Yonnet, J-P. and Latreche, M. E. H. (2009), "3D Analytical Calculation of Forces between Linear HalbachType Permanent Magnet Arrays", Proceedings of the 8th International Symposium on Advanced Electromechanical Motion Systems (ELECTROMOTION), Lille, France, pp. 382-387.

Cha, H-R., Cho, H-W. and Lee, S-H. (2008), “The Influence of Magnetization Pattern on the Performance of Permanent Magnet Eddy Current Couplings and Brakes", Journal of Electrical Engineering \& Technology, Vol. 3, No. 3, pp. 379-384.

Choi, J.-S. and Yoo, J. (2008), "Design of Halbach Magnet Array Based on Optimisation Techniques", IEEE Transaction on Magnetics, Vol. 44, No. 10, pp. 2361-2366.

Fan, J-J and Wu, J-H. (2012), "Optimization of Permanent Magnet Synchronous Motor with Halbach Magnet", Proceedings of the 2nd International Conference on Electronic \& Mechanical Engineering and Information Technology (EMEIT), Shenyang, Liaoning, China, pp. 1784-1788.

Halbach, K. (1980), "Design of permanent magnet multipole magnets with oriented rare earth cobalt material", Journal Nuclear Instruments and Methods, Vol. 169, No. 1, pp. 1-10.

Ibtissam, B., Mourad, M., Ammar, M. and Fouzi, G. (2014), "Magnetic Field Analysis of Halbach Permanent Magnetic Synchronous Machine", Proceedings of International Conference on Control, Engineering \& Information Technology (CEIT), Sousse, Tunisia, pp. 12-16.

Jian, L. and Chau, K. T. (2010), “A coaxial Magnetic Gear With Halbach Permanent-Magnet Array”, IEEE Transaction on Energy Conversion, Vol. 25, No. 2, pp. 319-328.

Kataoka, Y., Takayama, M., Matsushima, Y. and Anazawa, Y. (2013), "Comparison of Three Magnet Array-type Rotors in Surface Permanent Magnet-type Vernier Motor", Journal of International Conference on Electrical Machines and Systems (JICEMS), Vol. 2, No. 1, pp. 1-8.

Livadaru, L. et al. (2011), "Permanent Magnet Synchronous Machines with Halbach array Configurations - A F.E.M. Approach”, Buletinul AGIR, An. XVI, No. 4, pp. 17-22.

Mallinson, J. C. (1973), “One-sided Fluxes - A Magnetic Curiosity?”, IEEE Transaction on Magnetics, Vol. MAG-9, No. 4, pp. 678-682.

Mansson, D. (2015), "On the optimization of Halbach arrays as energy storage media", Journal Progress in Electromagnetics Research B, Vol. 62, pp. 277-288.

Mansson, D. (2014), "On the suitability of using Halbach arrays as potential energy storage media", Journal Progress in Electromagnetics Research B, Vol. 58, 151-166.

Mansson, D. (2015), "A Short Note on the Optimization of Halbach Arrays Used as Magnetic Springs", Progress In Electromagnetics Research Symposium Proceedings (PIERS), Prague, Czech Republic, pp. 1328-1332.

Marinescu, M. and Marinescu, N. (1992), "New concept of permanent magnet excitation for electrical machines Analytical and numerical computation", IEEE Transaction on Magnetics, Vol. 28, pp. 1390-1393.

Ofori-Tenkorang, J. and Lang, J. H. (1995), "A comparative analysis of torque production in Halbach and conventional surface-mounted permanent-magnet synchronous motors", Proceedings of the 30th IEEE Industry Applications Conference (IAS), Orlando, Florida, USA, pp. 657-663.

Petkovska, L., Cvetkovski, G. and Lefley, P. (2015), "Analysis of the stator topology impact on cogging torque for surface permanent magnet motor", COMPEL: The International Journal for Computation and Mathematics in Electrical and Electronic Engineering, Vol. 34, Issue 2, pp. 456-474.

Rovers, J. M. M., J. W. Jansen, E. A. Lomonova, and M. J. C. Ronde (2009), "Calculation of the static forces among the permanent magnets in a Halbach array”, IEEE Transaction on Magnetics, Vol. 45, No. 10, pp. 4372 - 4375.

Sadeghi, S. and Parsa, L. (2012), "Improved technique for minimizing torque pulsation in Halbach array permanent magnet machines", COMPEL: The International Journal for Computation and Mathematics in Electrical and Electronic Engineering, Vol. 31 No. 6, pp. 1590-1602.

Shute, H. A., Mallinson, J. C., Wilton, D. T. and Mapps, D. J. (2000), "One-sided fluxes in planar, cylindrical, and spherical magnetized structures", IEEE Transaction on Magnetics, Vol. 36, No. 2, pp. 440-451.

Winter, O., Kral, C. and Schmidt, E. (2012), "Design study of magnet shapes for axial Halbach arrays using 3D Finite Element Analyses", Proceedings of XXth International conference on Electrical Machines (ICEM), Marseille, France, pp. 2660-2665.

Zhu, Z. Q., Xia, Z. P. and Howe, D. (2002), "Comparison of Halbach Magnetized Brushless Machines Based on Discrete Magnet Segments or a Single Ring Magnet”, IEEE Transaction on Magnetics, Vol. 38, No. 5, pp. 2997 2999. 